\title{
Machine-Learned Molecular Surface and Its Application to Implicit Solvent Simulations
}

\author{
Haixin Wei, Zekai Zhao, and Ray Luo \\ Departments of Materials Science and Engineering, Molecular Biology and Biochemistry, Chemical and \\ Biomolecular Engineering, and Biomedical Engineering, Graduate Program in Chemical and Materials \\ Physics, University of California, Irvine, California 92697, United States
}




\section{Supplemental Methods}

\section{Training Details}

The training was conducted in three steps. First, the model was trained incrementally by adding one atom at a time until all the nearby atoms are fed into the model to complete the training as shown in Figure 2 and Figure S1. This step is necessary to initialize the ANN model training, because for such a high-dimension (200 features) nonlinear system, its local minimum can be extremely deep, causing the training optimization to fail.

The second step in the training process was to determine how many neurons are needed for the hidden layers. To determine the neurons needed in the first hidden layer, the number of neurons of both layers are varied, and the results are shown in Figure S2(a). Clearly, for the three tested training sets, all show that 100-neurons perform the best. Next, the first hidden layer was kept at 100 neurons, and the second hidden layer was varied, and the analysis is shown in Figure S2(b). Figure S2(b) shows that all tested conditions perform similarly, with 40 neurons slightly better than others so 40 neurons were selected.

The third step was to finalize the model. Given the model structure, which contains two hidden layers, with 100 neurons and 40 neurons respectively, we trained the model with all the training sets. To minimize GPU memory usage, the model was trained sequentially with one training set at a time. For the first training set, the model was trained incrementally by adding one atom at a time as discussed above. After the first training set, all nearby atoms were fed to the model in the subsequent training of remaining five sets. The training order among the training sets is irrelevant, because all training sets perform extremely similarly (for example Figures 2 v.s. Figure S1 and Figure S2). 


\section{Supplemental Figures}

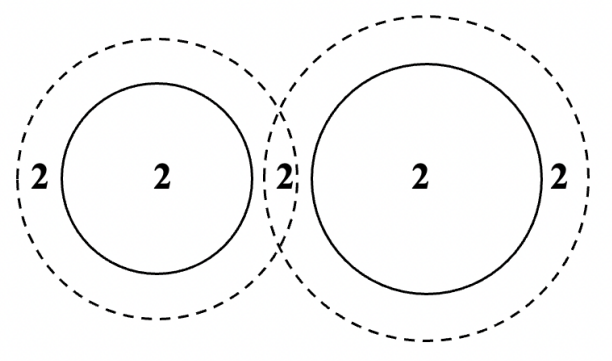

Step 1

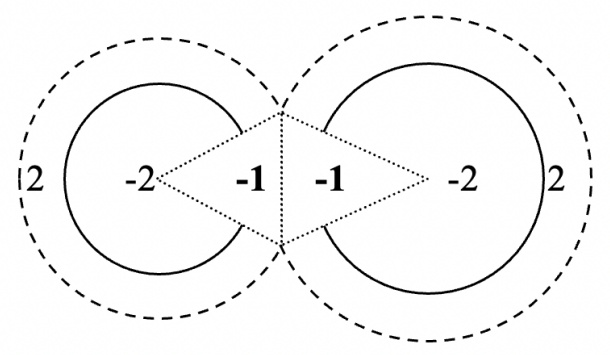

Step 3

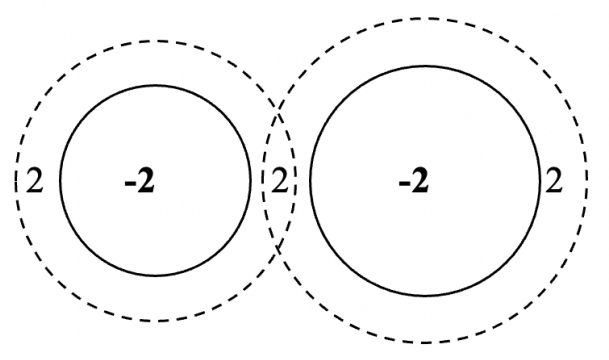

Step 2

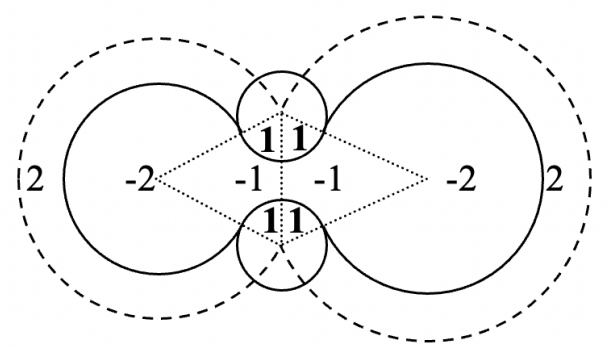

Step 4

Figure S1. Grid point labeling scheme in the Amber/PBSA classical SES surface builder. There are four steps in the labeling scheme. 1. Label all grid points within SAS (coarse dashed circles) as "2". 2. Label all grid points within VDW as "-2". 3. Label all grid points within the reentry cones (fine dashed lines) of all pairs of neighboring atoms as "-1". 4. Label all "-1" grid points within any solvent probe sphere at a solvent accessible arc dot as "1". After labeling, all exterior grid points are labeled with positive integers and all interior grid points are labeled with negative integers. Note that the signs of training data used in this study is the opposite of the signs used in the labeling scheme, to be consistent with the convention in the level set function. Specifically, the outside solvent region is positive and the inside solute region is negative in Amber/PBSA, but the outside region is negative and the inside region is positive in the level set function. 

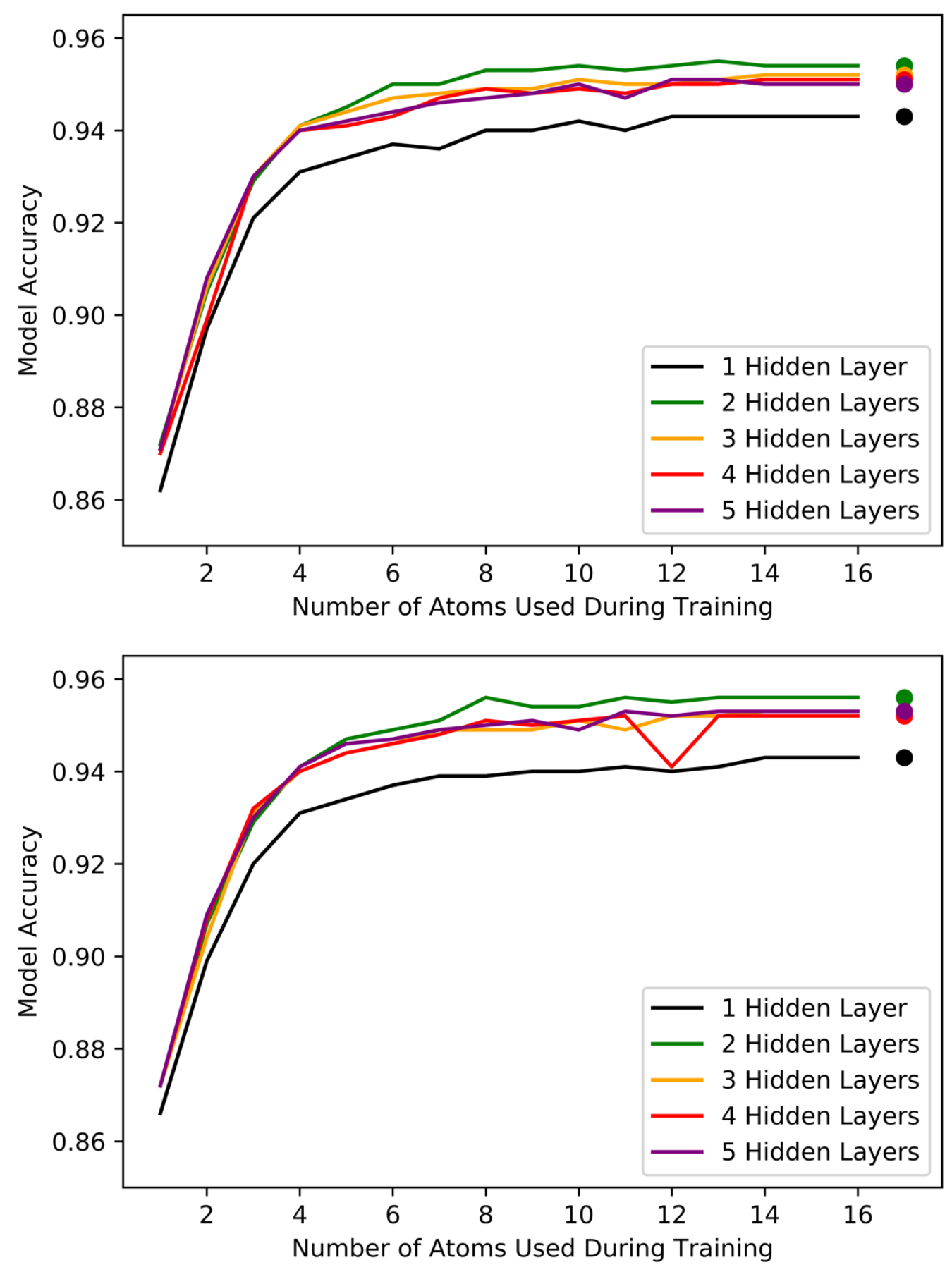

Figure S2. Incremental training of the model for the second and the third data set. The ANN model was set up with different numbers of hidden layers, each hidden layer is with 200 neurons. 


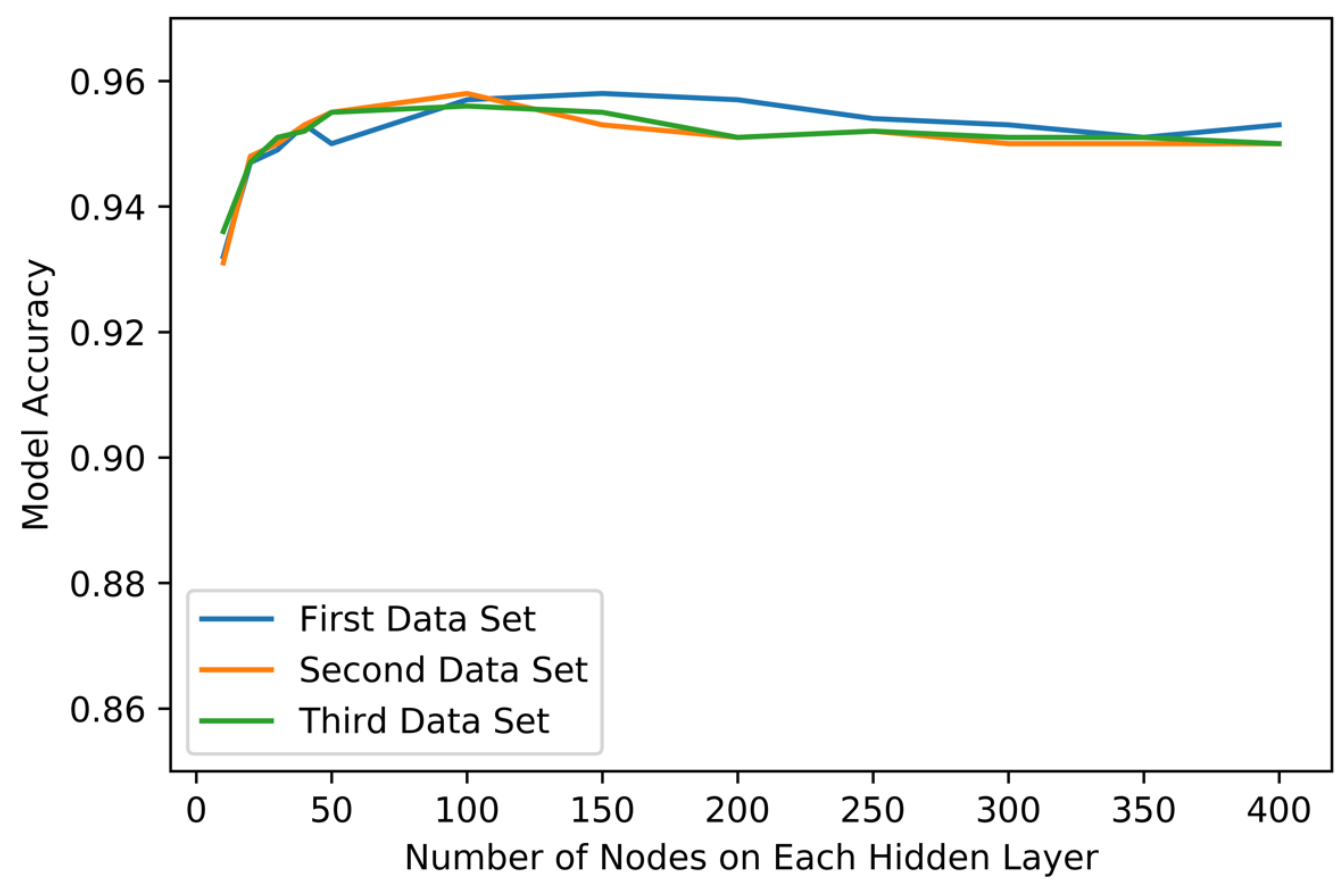

(a)

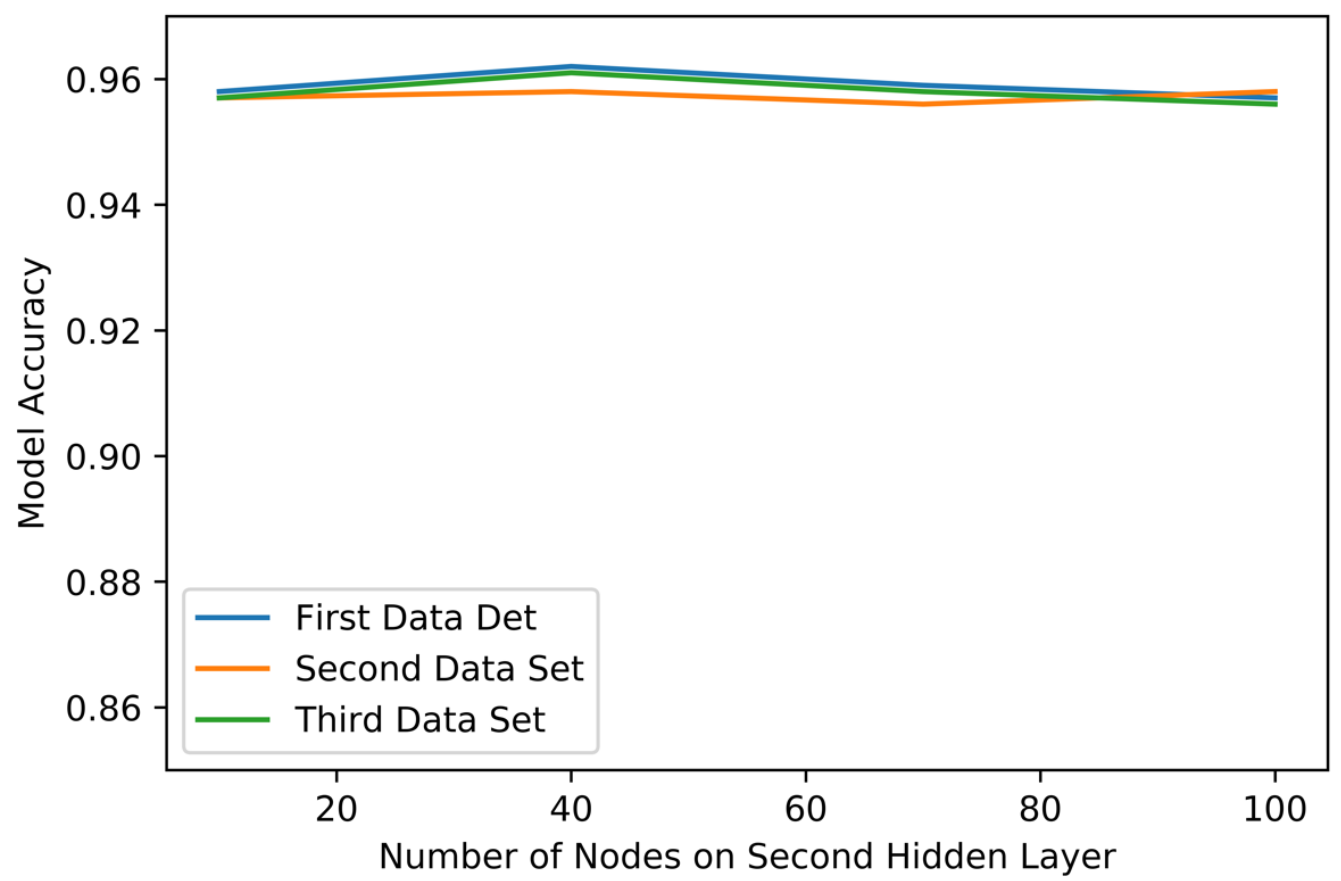

(b)

Figure S3. Numbers of neurons needed in the two hidden layers versus model accuracy. (a) First layer analysis in the top panel. (b) Second layer analysis in the bottom panel. 


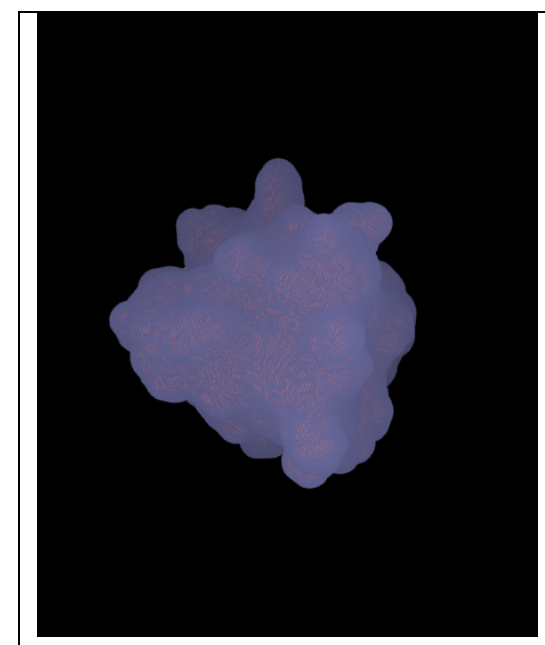

(a)

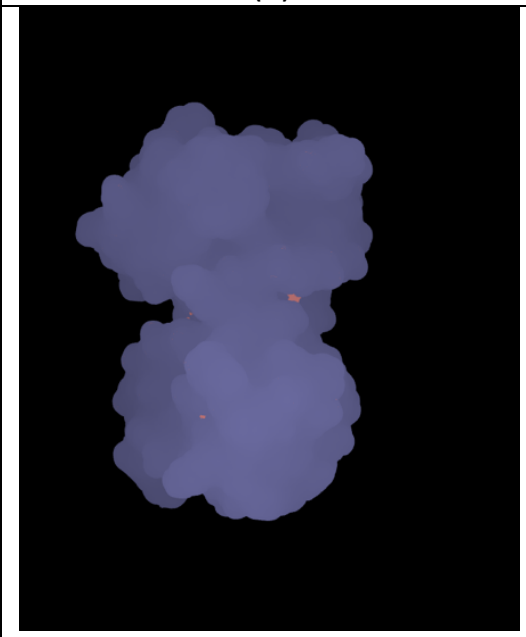

(d)

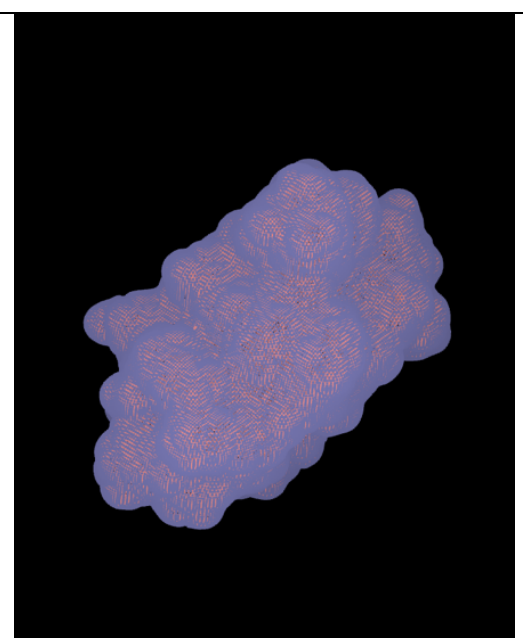

(b)

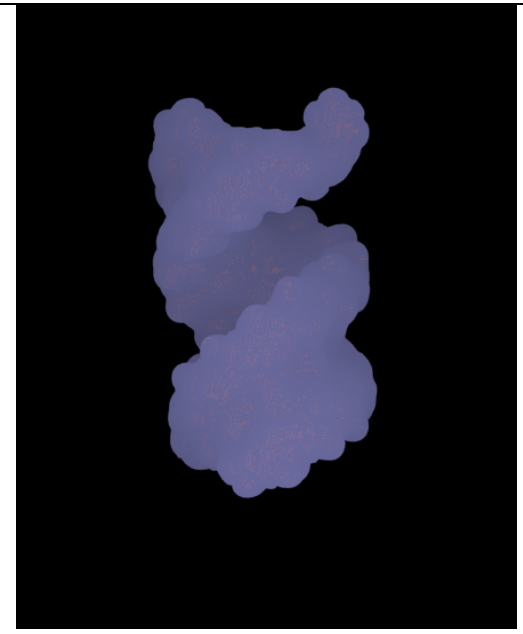

(e)

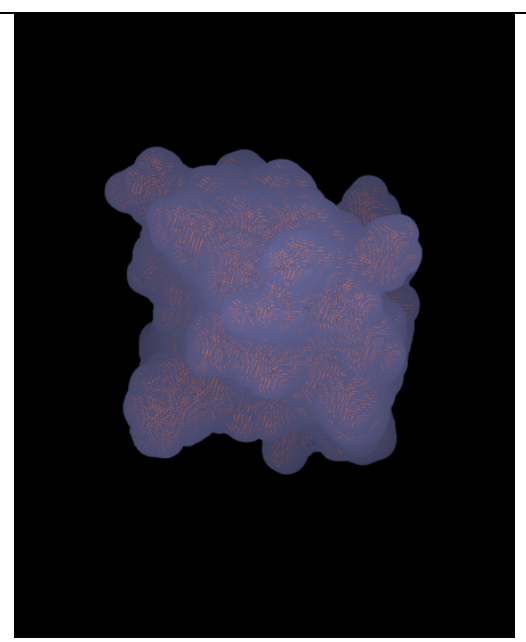

(c)

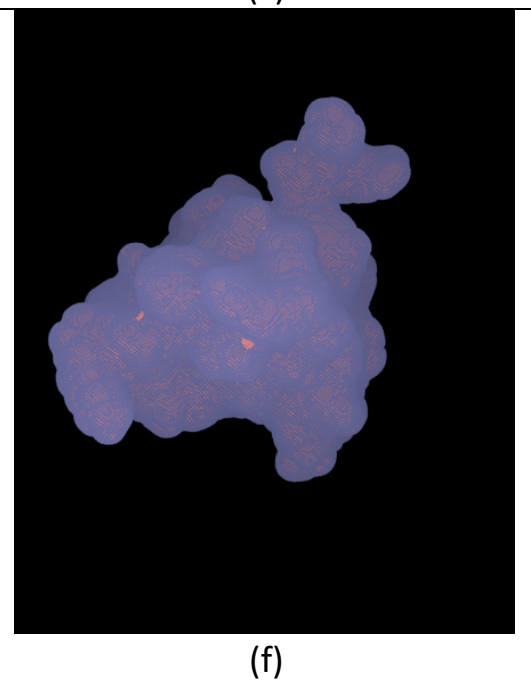

(f)

Figure S4. Superimposed rendering of density function surface (blue) and the classical SES (red) of representative molecules. (a) PDB ID: 1enh, all-alpha protein; (b) PDB ID: 1pgb, all-beta protein; (c) PDB ID: 1shg, alpha/beta protein; (d) PDB ID: 1w0u, protein/protein complex; (e) PDB ID: 3czw, RNA duplex; (f) PDB ID: 3fdt, protein/DNA complex. 


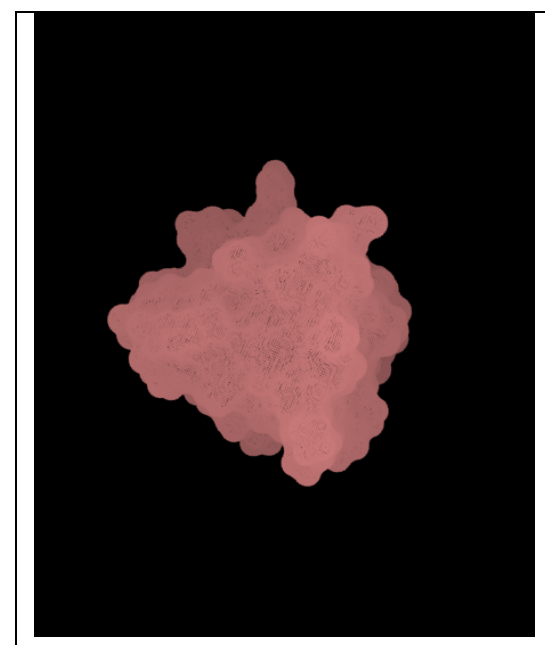

(a)

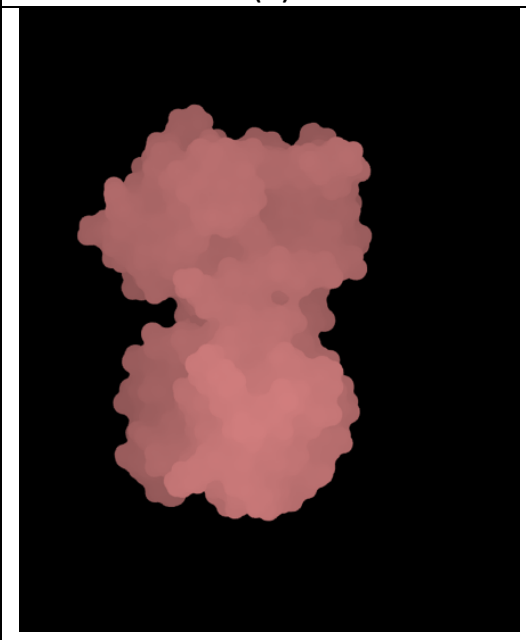

(d)

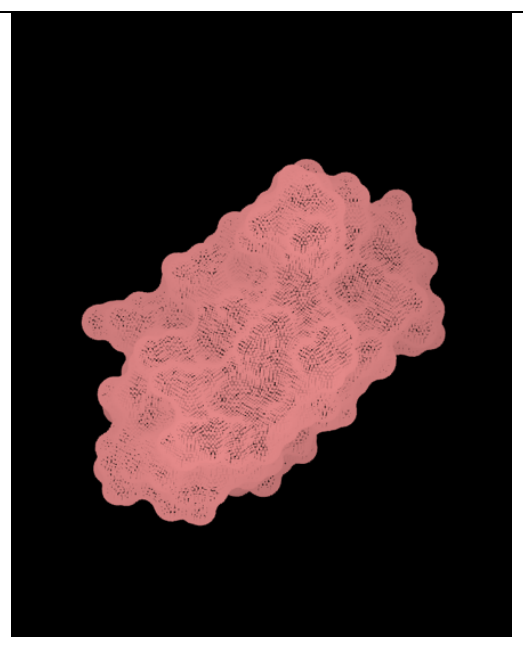

(b)

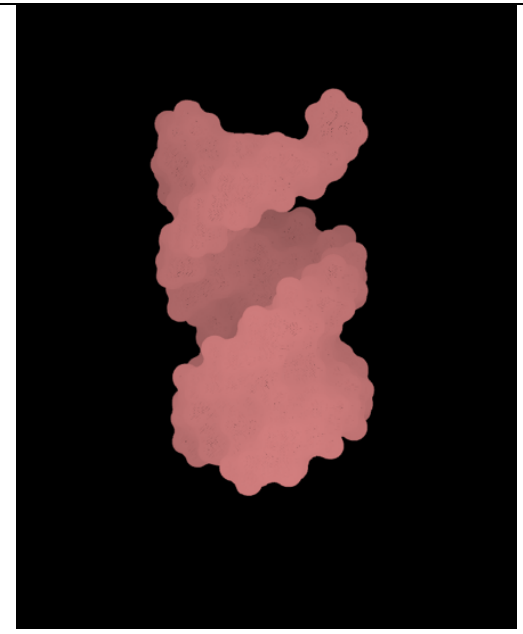

(e)

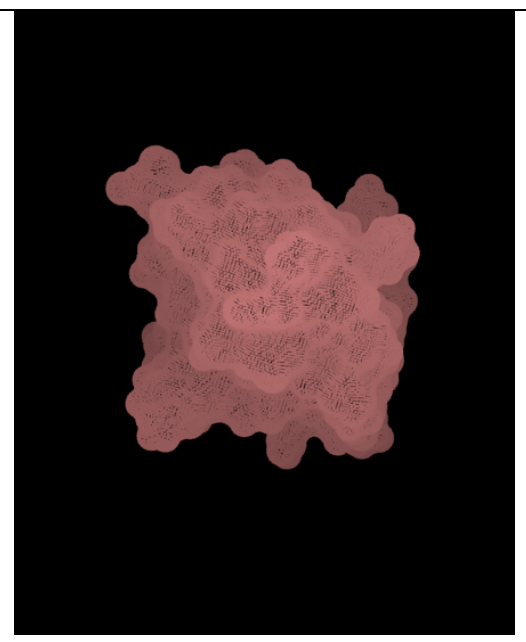

(c)

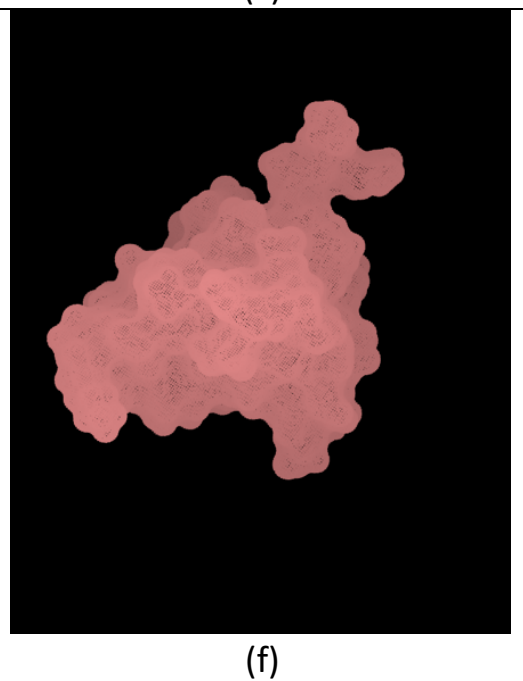

(f)

Figure S5. Rending of classical SES of representative molecules. (a) PDB ID: 1enh, all-alpha protein; (b) PDB ID: 1pgb, all-beta protein; (c) PDB ID: 1shg, alpha/beta protein; (d) PDB ID: 1w0u, protein/protein complex; (e) PDB ID: 3czw, RNA duplex; (f) PDB ID: 3fdt, protein/DNA complex. 


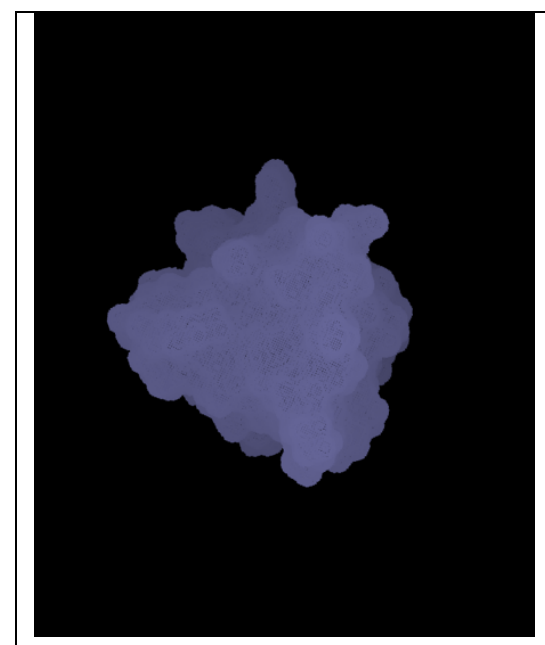

(a)

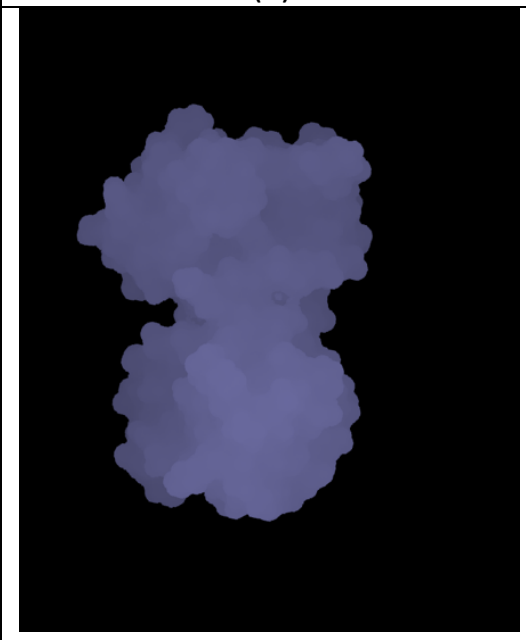

(d)

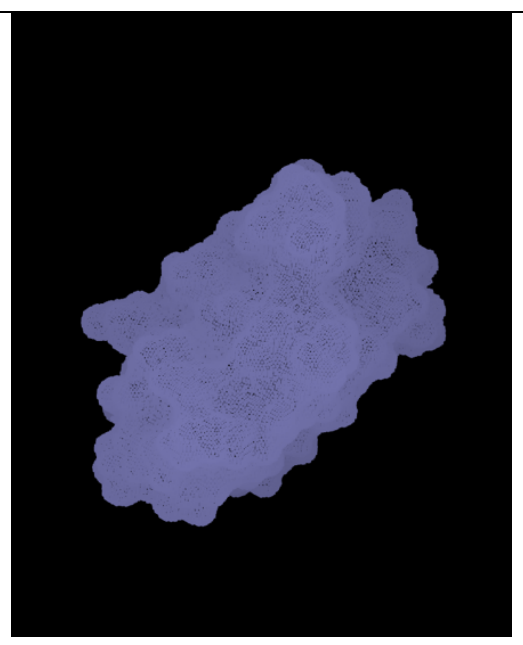

(b)

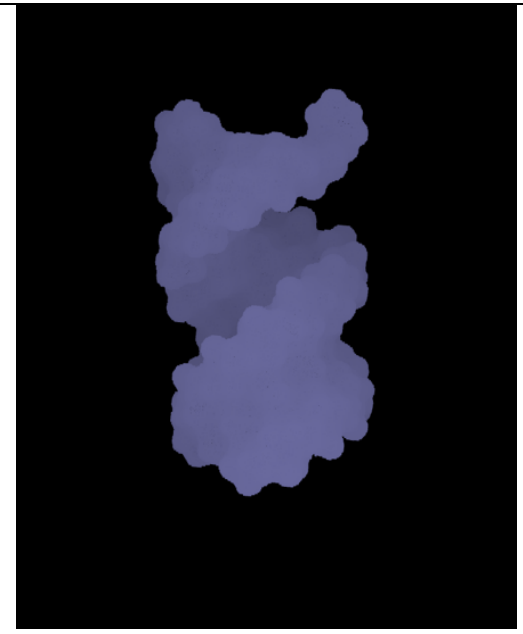

(e)

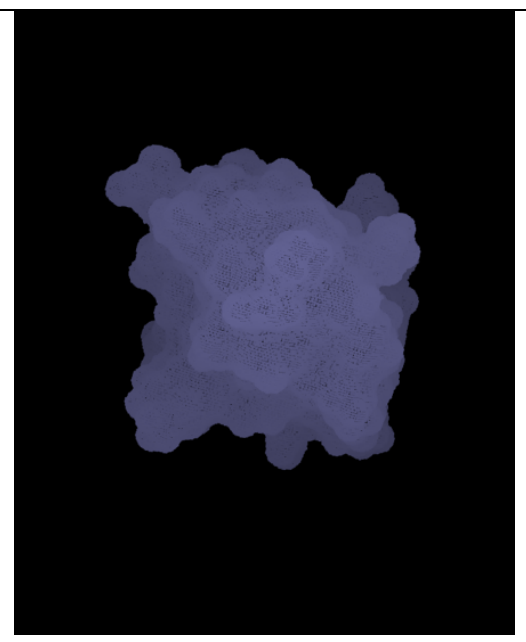

(c)

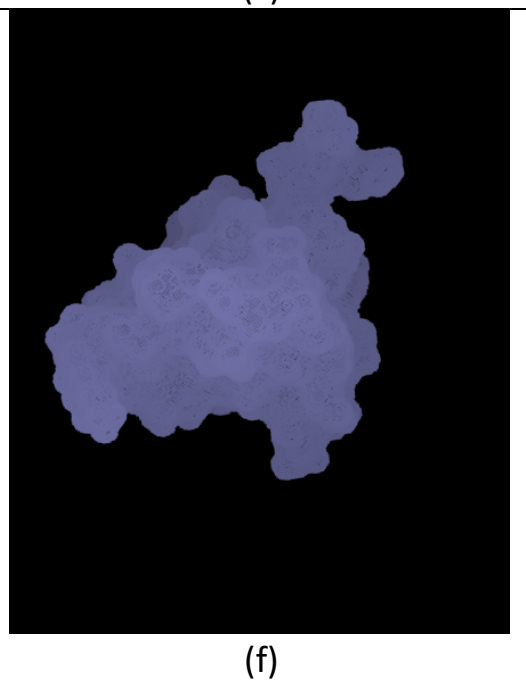

(f)

Figure S6. Rending of machine-learned SES of representative molecules. (a) PDB ID: 1enh, all-alpha protein; (b) PDB ID: 1pgb, all-beta protein; (c) PDB ID: 1shg, alpha/beta protein; (d) PDB ID: 1w0u, protein/protein complex; (e) PDB ID: 3czw, RNA duplex; (f) PDB ID: 3fdt, protein/DNA complex. 


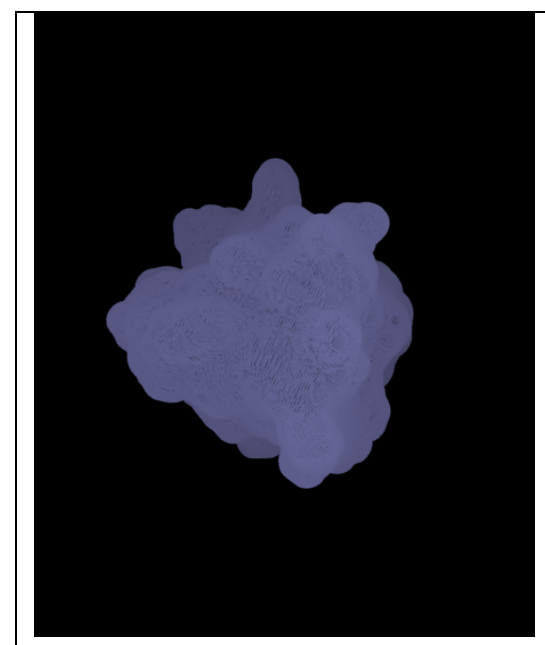

(a)

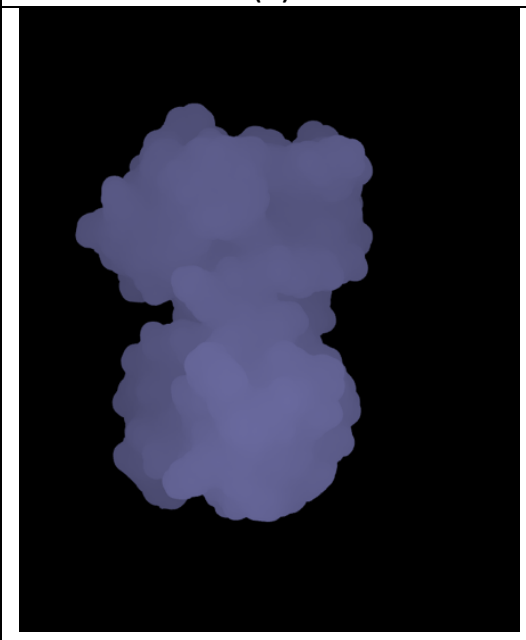

(d)

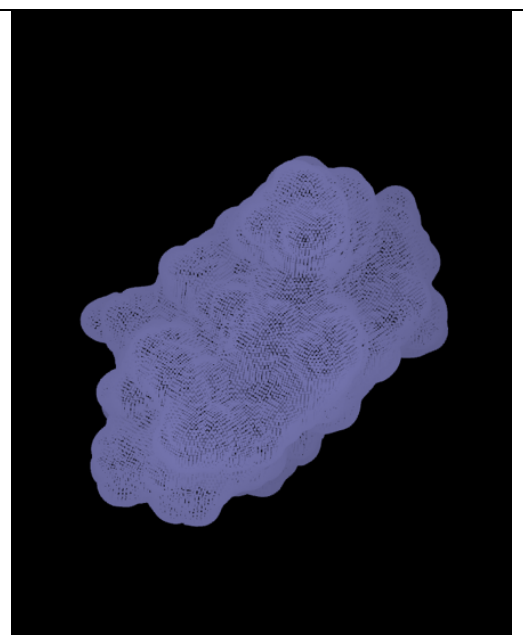

(b)

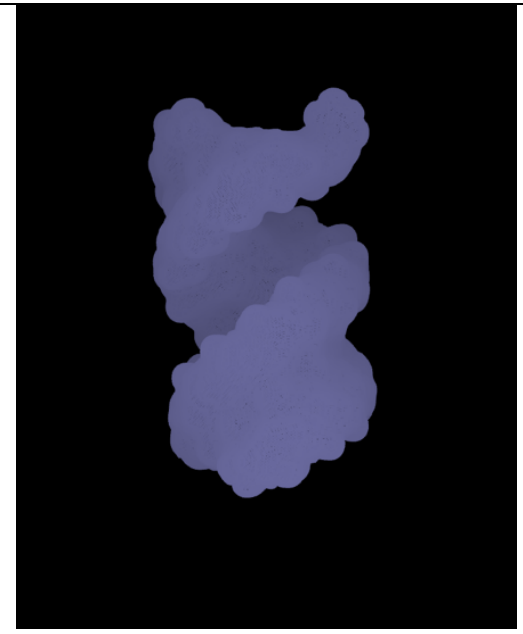

(e)

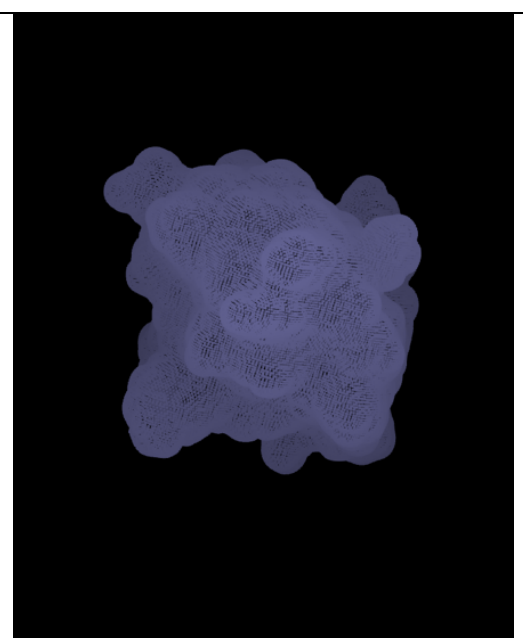

(c)

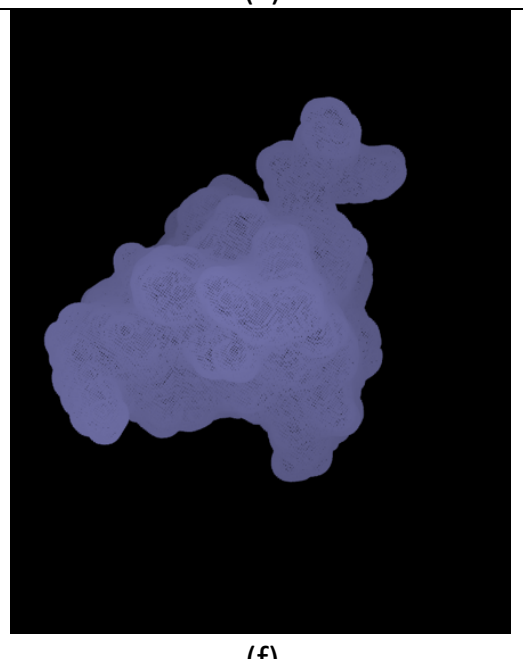

(f)

Figure S7. Rending of density function surface of representative molecules. (a) PDB ID: 1enh, all-alpha protein; (b) PDB ID: 1pgb, all-beta protein; (c) PDB ID: 1shg, alpha/beta protein; (d) PDB ID: 1w0u, protein/protein complex; (e) PDB ID: 3czw, RNA duplex; (f) PDB ID: 3fdt, protein/DNA complex. 


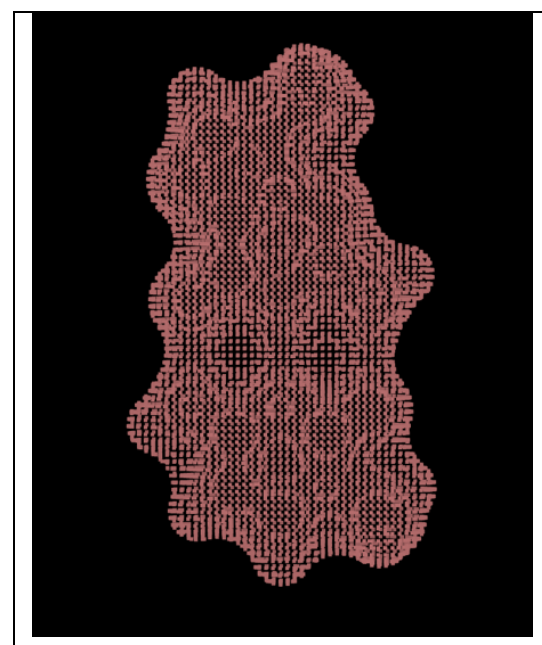

(a)

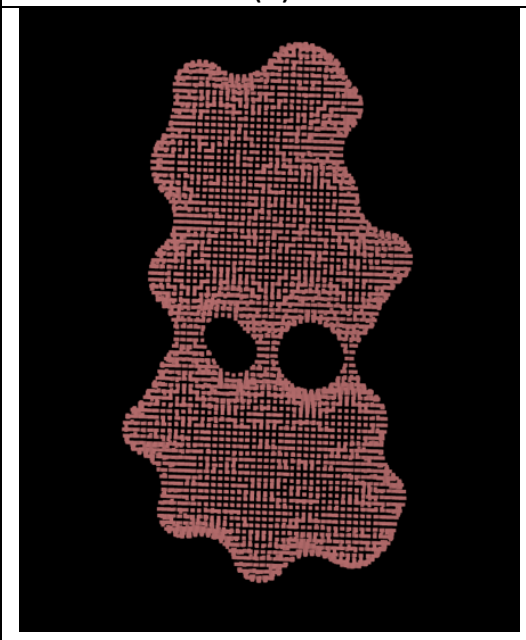

(d)

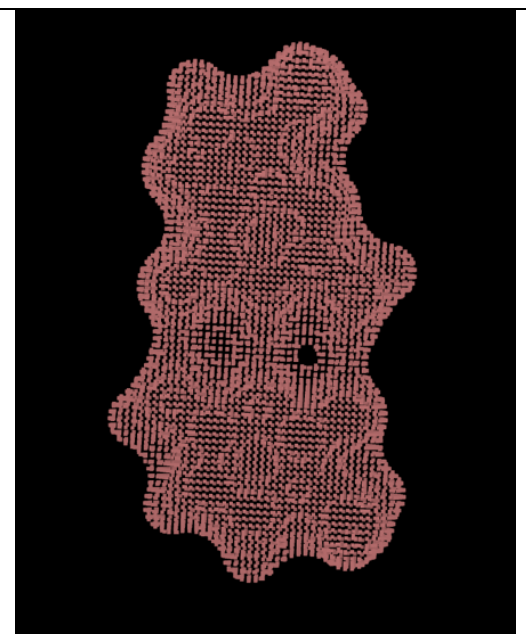

(b)

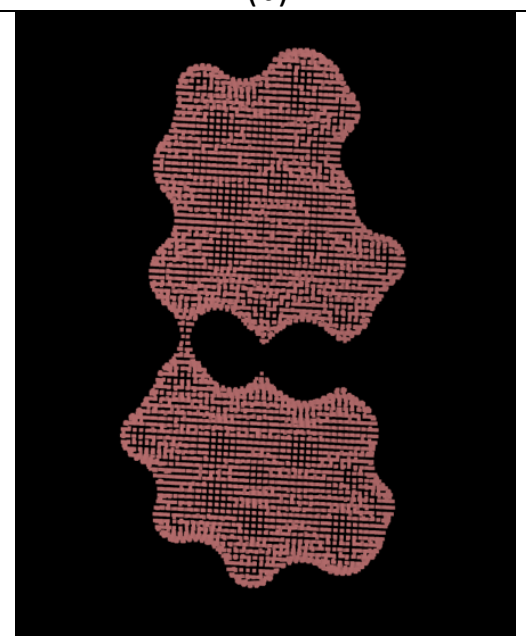

(e)

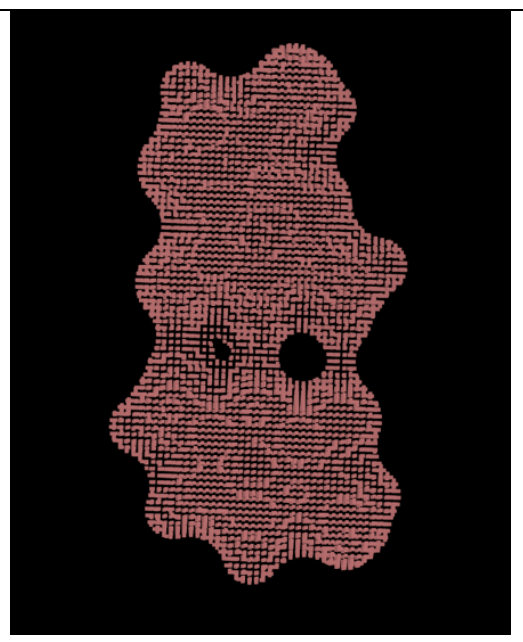

(c)

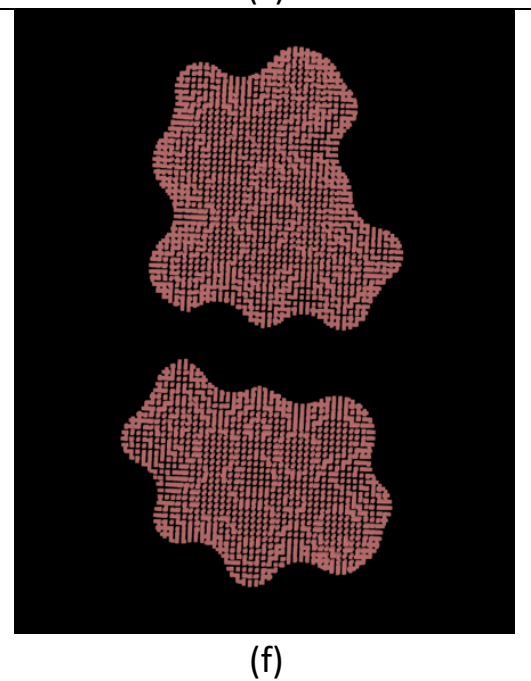

Figure S8. Rendering of classical SES of the GC complex. The two molecules are manually pulled apart by $0.5 \AA ̊$ each step. 


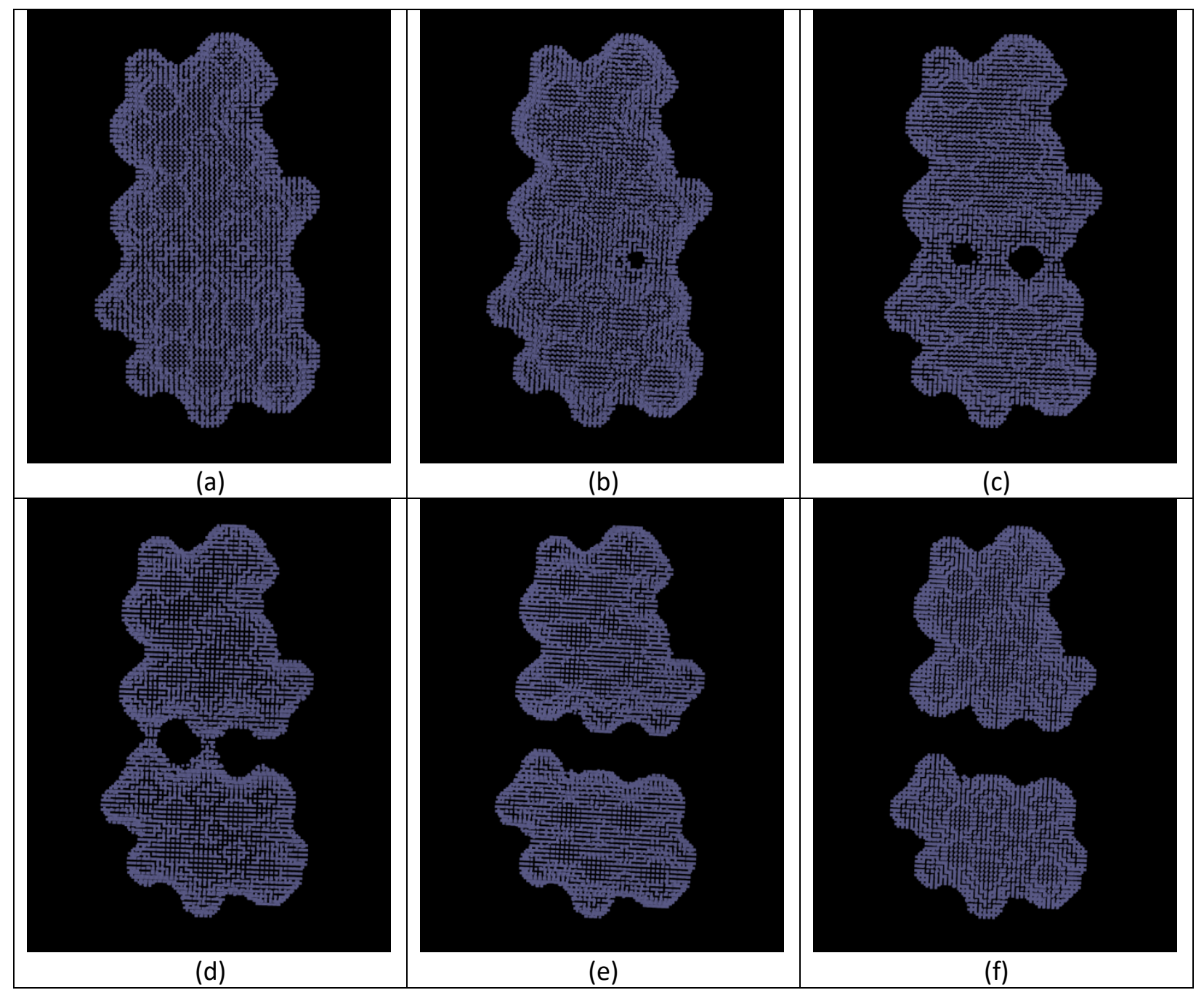

Figure S9. Rendering of machine-learned SES of the GC complex. The two molecules are manually pulled apart by $0.5 \AA$ A each step. 


\section{Supplemental Movies}

Set 1. Animated renderings of superimposed machine-learned SES and classical SES for (a) PDB ID: 1enh, all-alpha protein; (b) PDB ID: 1pgb, all-beta protein; (c) PDB ID: 1shg, alpha/beta protein; (d) PDB ID:

1w0u, protein/protein complex; (e) PDB ID: 3czw, RNA duplex; (f) PDB ID: 3fdt, protein/DNA complex.

Set 2. Animated renderings of superimposed density function surface and classical SES for (a) PDB ID: 1enh, all-alpha protein; (b) PDB ID: 1pgb, all-beta protein; (c) PDB ID: 1shg, alpha/beta protein; (d) PDB ID: 1w0u, protein/protein complex; (e) PDB ID: 3czw, RNA duplex; (f) PDB ID: 3fdt, protein/DNA complex. 